\title{
Synthesis and characterization of electrospun polyvinyl alcohol nanofibrous scaffolds modified by blending with chitosan for neural tissue engineering
}

\author{
This article was published in the following Dove Press journal: \\ International Journal of Nanomedicine \\ 28 December 2011 \\ Number of times this article has been viewed
}

\author{
Sanaz Naghavi Alhosseini' \\ Fathollah Moztarzadeh' \\ Masoud Mozafari' \\ Shadnaz Asgari \\ Masumeh Dodel ${ }^{3}$ \\ Ali Samadikuchaksaraei ${ }^{4,5}$ \\ Saeid Kargozar 6 \\ Newsha Jalali' \\ 'Biomaterials Group, Faculty of \\ Biomedical Engineering (Center of \\ Excellence), Amirkabir University \\ of Technology, Tehran, Iran; ${ }^{2}$ Neural \\ Systems and Dynamics Laboratory, \\ Department of Neurosurgery, \\ David Geffen School of Medicine, \\ University of California, Los Angeles, \\ CA, USA; ${ }^{3}$ Nanotechnology and Tissue \\ Engineering Department, Stem Cell \\ Technology Research Center, Tehran, \\ Iran; ${ }^{4}$ Department of Biotechnology \\ and Cellular and Molecular Research \\ Center, Tehran University of Medical \\ Sciences, Tehran, Iran; ${ }^{5}$ Biological \\ Systems Engineering Laboratory, \\ Centre for Process Systems \\ Engineering, Department of Chemical \\ Engineering, Imperial College \\ London, London, UK; ${ }^{6}$ Department of \\ Biotechnology, Tehran University of \\ Medical Sciences, Tehran, Iran
}

Correspondence: Masoud Mozafari Biomaterials Group, Faculty of Biomedical Engineering (Center of Excellence), Amirkabir University of Technology, PO Box 15875-44I 3, Tehran, Iran Tel +98-2I 22354I62

Fax +98-2I 223737।

Email mmozafari@aut.ac.ir

\begin{abstract}
Among several attempts to integrate tissue engineering concepts into strategies to repair different parts of the human body, neuronal repair stands as a challenging area due to the complexity of the structure and function of the nervous system and the low efficiency of conventional repair approaches. Herein, electrospun polyvinyl alcohol (PVA)/chitosan nanofibrous scaffolds have been synthesized with large pore sizes as potential matrices for nervous tissue engineering and repair. PVA fibers were modified through blending with chitosan and porosity of scaffolds was measured at various levels of their depth through an image analysis method. In addition, the structural, physicochemical, biodegradability, and swelling of the chitosan nanofibrous scaffolds were evaluated. The chitosan-containing scaffolds were used for in vitro cell culture in contact with PC12 nerve cells, and they were found to exhibit the most balanced properties to meet the basic required specifications for nerve cells. It could be concluded that addition of chitosan to the PVA scaffolds enhances viability and proliferation of nerve cells, which increases the biocompatibility of the scaffolds. In fact, addition of a small percentage of chitosan to the PVA scaffolds proved to be a promising approach for synthesis of a neural-friendly polymeric blend.
\end{abstract}

Keywords: polyvinyl alcohol, chitosan, polymer blending, nanofibrous scaffolds, neural tissue engineering

\section{Introduction}

Generally speaking, the chemical composition and structure of tissue engineering scaffolds should be optimized for supporting the reparative process in a particular tissue and for attachment and proliferation of particular cells. ${ }^{1}$ Among different available scaffolds, those recently developed with nanofibrous structures seem to be promising substrates for tissue engineering applications due to their high structural similarity to native extracellular matrix..$^{2-5}$

Fiber-based porous scaffolds, which structurally mimic the extracellular matrix (ECM), have been synthesized from numerous natural or synthetic biopolymers. These scaffolds have been specifically engineered by electrospinning platform technology, and were successfully used for nerve tissue engineering applications. Electrospun scaffolds could be optimized to closely mimic the chemical, physical, and biological properties of the extracellular matrix of a particular tissue by fine-tuning its fabrication method and modifying the components of the composites. For most tissues, the biological properties of the scaffolds, including promotion of cell adhesion, proliferation, and 
differentiation, are more important than its microstructure. However, for neural tissues, the microstructure of the scaffold, especially its three-dimensionality, is equally as important as its biological properties. ${ }^{6-9}$

Electrospinning is a unique technology which can produce non-woven fibrous structures with fiber diameters ranging from nanometers to microns. This range of fiber size is difficult to achieve by other fabrication methods. ${ }^{10,11}$ The scaffolds fabricated by these nanofibers possess an extremely high ratio of surface to volume, have adjustable porosity, and could easily be customized over a wide range of sizes, shapes, and mechanical properties, which makes them very suitable candidates for neural tissue engineering. ${ }^{12,13}$ In electrospinning, polymer blending is one of the most effective methods for preparation of composites with specific properties. ${ }^{14}$ The key issue in this method is adjustment of the ratio of the components, which influences the diameter and morphology of the structure of the fibers and scaffolds and the biological properties of the scaffolds. ${ }^{15}$ Fine-tuning of nanostructured topographical cues such as grooves, ridges, pores, and nodes is also important as they influence cell adhesion, migration, proliferation, and differentiation. ${ }^{5}$

So far, neural scaffolds have been synthesized from several natural and synthetic materials using different fabrication techniques, including electrospinning. The electrospun biodegradable polymers were successfully tested for their efficacy to stimulate axonal regeneration and neural stem cell differentiation, taking into account their different structural properties such as the diameter and alignment of the nanofibers. ${ }^{16-22}$ The biodegradable polymers used for this purpose mostly include polylactic-co-glycolic acid (PLGA), polyvinyl alcohol (PVA), collagen, and chitosan..$^{23,24}$ Of these, PVA is a non-toxic, hydrophilic, and biocompatible material which has also been used for other tissue engineering applications. ${ }^{25,26}$ Chitosan has been widely used in this field as well. ${ }^{27,28}$ However, most of the previous studies focus on a single polymer for fabrication of electrospun nanofibrous scaffolds.

The high biocompatibility of chitosan has led several groups to use different techniques for scaffold fabrication, such as different three-dimensional shapes including tubular conduits, using chitosan as the base material. ${ }^{29,30}$ However, the mechanical properties of these scaffolds are still not optimal for application in nervous tissue. It has been shown that chitosan has quite positive effects on nerve tissue regeneration. ${ }^{31-33}$ Here, we tried to modify the physicochemical and biological properties of PVA polymer by blending it with chitosan, and used electrospinning for fabrication of a neurocompatible scaffold.

\section{Materials and methods \\ Materials}

PVA (98\% hydrolyzed, average molecular weight of $72000 \mathrm{gmol}^{-1}$ ), acetic acid (AA), and glutaraldehyde (GA) (25\% aqueous solution) were purchased from Merck (Darmstadt, Germany). Chitosan (medium molecular weight of 190,000-310,000) was purchased from Orbital Pharma Co (Hebei, China).

\section{Electrospinning procedures}

The electrospinning setup utilized in this study consisted of an adjustable high DC voltage power supply, two syringe pumps (SP-500; JMS, Tokyo, Japan) and a ground electrode (a stainless steel drum with an external diameter of $50 \mathrm{~mm}$, length of $10 \mathrm{~cm}$, and variable rotating speed). Polymer solution was placed into metal capillaries (an internal diameter of $0.8 \mathrm{~mm}$ and length of $20 \mathrm{~mm}$ ) with a constant mass flow rate of $0.6 \mathrm{~mL} /$ hour. PVA was dissolved in distilled water (DW) at a concentration of $10 \mathrm{wt} \%$ and chitosan was dissolved in acetic acid-water (AA-water) solution (2 wt $\%$ ) at a concentration of $2 \mathrm{wt} \%$. The PVA-DW solution $10 \mathrm{wt} \%$ was mixed with the chitosan-AA solution $2 \mathrm{wt} \%$ at a weight ratio of (PVA/chitosan) 90/10. Then, the mixed solution and PVA-DW solution (10 wt $\%$ ) were subjected to the electrospinning experiment separately. The distance between the tips and the ground electrode was $15 \mathrm{~cm}$, while the positive voltage applied to the polymer solutions was $20 \mathrm{kV}$. The drum was continuously rotating at $250 \mathrm{rpm}$ throughout the course of electrospinning.

Finally, samples were soaked in a cross-linking bath with GA vapor for 24 hours to cross-link the polymeric chains, reduce degradation, and enhance the biomechanical properties of the scaffolds for tissue repair. After soaking in the bath, the samples were carefully washed with $2 \%$ glycine aqueous solution several times to remove the remaining amount of GA.

\section{Characterization}

\section{Viscosity measurement}

Before the electrospinning process, the viscosity of solutions was measured by Brookfield Model DV-III viscometer (Brookfield Engineering Laboratories Inc, Stoughton, MA).

\section{Morphology and microstructure analysis}

The morphology and microstructure of the synthesized samples were evaluated using SEM. The electrospun fiber samples were coated with a thin layer of gold $(\mathrm{Au})$ by sputtering (Emitech K450X, Ashford, UK) and their 
morphologies were observed under a scanning electron microscope (AIS2100; Seron Technology, Uiwang-si, Gyeonggi-do, South Korea) that operated at the acceleration voltage of $15 \mathrm{kV}$.

Image analysis program ImageJ (US National Institute of Health, Bethesda, MD), which uses grayscale level processing based on image structure, ${ }^{34}$ was used to characterize the SEM micrographs in the original magnification of $30 \times$. The average diameters of fibers and the porosity of various layers were calculated by this software package. Using different thresholds, the SEM micrographs were converted to binary images, and porosity of scaffolds were calculated in various layers by a recently developed method described by Ghasemi-Mobarakeh et al. ${ }^{35}$

\section{FTIR analysis}

The samples were examined by Fourier transform infrared (FTIR) analysis with a Nicolet 17DSX FT-IR spectrometer (Thermo Scientific, Waltham, MA). For IR analysis, $1 \mathrm{mg}$ of the scraped samples was carefully mixed with $300 \mathrm{mg}$ of $\mathrm{KBr}$ (infrared grade) and pelletized under vacuum. Then, pellets between 500 and $4000 \mathrm{~cm}^{-1}$ were analyzed with 120 scans averaging $4 \mathrm{~cm}^{-1}$ resolution. The FTIR analysis was used to characterize the presence of specific chemical groups of PVA and chitosan.

\section{Swelling test}

The prepared electrospun nanofibrous scaffolds were placed in a 24-well plate. Each well contained $1 \mathrm{~mL}$ of a phosphate buffered solution (PBS; pH 7.4). The scaffolds were incubated in vitro at $37^{\circ} \mathrm{C}$ for different periods of time $(1,3,7$, and 10 days).${ }^{36}$ After immersion of the scaffolds in PBS solution for these different periods, the amount of fluid uptake was determined by careful removal of samples from the medium after wiping off excess fluid with filter paper. The swelling value $(S)$ was calculated using equation 1 :

$$
S=\left(W_{w}-W_{d}\right) / W_{d} \times 100
$$

For this test the samples were weighed for determination of the wet weight $\left(W_{w}\right)$ as a function of immersion time. $W_{d}$ is the dried weight of the samples.

\section{Degradation test}

The degradation study of the scaffolds was carried out in vitro by incubating the samples in $\mathrm{PBS}$ at $\mathrm{pH} 7.4,37^{\circ} \mathrm{C}$ for different periods of time. After each degradation period, the samples were washed and subsequently dried in a vacuum oven at room temperature for 24 hours. In order to find out the degradation index $\left(D_{i}\right)$, the weight of the samples $\left(W_{t}\right)$ and the degradation index was calculated based on the mass loss using equation 2 :

$$
D_{i}=\left(W_{0}-W_{t}\right) / W_{0} \times 100
$$

In vitro study in contact with PCI 2 nerve cell line The in vitro cytotoxicity of the prepared scaffolds was tested using the PC12 nerve cell line. The line was kept in continuous culture in Dulbecco's Modified Eagle Medium (DMEM) supplemented with $10 \%$ fetal bovine serum (FBS) and streptomycin/penicillin $100 \mathrm{U} / \mathrm{mL}$ (1\%). The cells were detached with trypsin/EDTA before seeding on samples. For seeding, the cells were trypsinized, centrifuged, and resuspended in complete culture medium. In the 24 -well plate, 90,000 cells were seeded in each well. Then, $6 \mathrm{mg} / \mathrm{mL}$ of synthesized scaffolds were added to each well. After 72 hours of incubation, MTT solution (5 mg/mL) (Sigma, Munich, Germany) was added into each well and incubated for 90-120 minutes.

Then, all the media was discarded and $600 \mu \mathrm{L}$ DMSO was added to each well. The OD (optical density) values were measured after 30 minutes by an ELISA reader at $590 \mathrm{~nm}$ with a reference filter of $620 \mathrm{~nm}$.

For scanning electron microscopy (SEM), the cells were harvested after 3 days of culture. The samples were fixed with 3\% GA for 2 hours. Specimens were rinsed in water and dehydrated with graded concentrations $(50 \%, 70 \%, 90 \%$, $100 \% \mathrm{v} / \mathrm{v}$ ) of ethanol. Subsequently, the samples were treated with hexamethyldisilazane (HMDS) and kept in a fume hood for air drying. Finally, the samples were coated with gold to observe cell morphology.

\section{Statistical analysis}

All experiments were performed in fifth replicate. The results were given as mean \pm standard error (SE). Statistical analysis was performed by one-way ANOVA and Tukey's test, with significance reported when $P<0.05$. The KolmogorovSmirnov test was used for assessment of distribution of data.

\section{Results and discussion Viscosity behavior}

Before electrospinning, the viscosities of the solutions were measured by Brookfield Model DV-III viscometer. The viscosity of PVA solution was 557 centipoise and that of PVA/chitosan solution (with the weight ratio of 90/10) was 1726 centipoise. This is in agreement with the data published by Paipitak et $\mathrm{al}^{37}$ who reported a linear increase in viscosity 
of PVA solution after blending with increasing amounts of chitosan. The high viscosity increases the interaction of two polymers, mainly through hydrogen bonding, and decreases the effects of surface tension. This will result in formation of fibers with uniform morphology after electrospinning. ${ }^{3}$

In recent years, polymer blending has become a method for providing polymeric materials with desirable properties for practical applications. In particular, chitosan blended with PVA has been reported to have good mechanical and chemical properties and, as a topic of great interest, has been extensively studied in the biomedical field. ${ }^{38-40}$ The enhanced property has been attributed to the interactions between chitosan and PVA in the blend through hydrophobic side-chain aggregation and intermolecular and intra-molecular hydrogen bonds $\mathrm{s}^{41,42}$ as shown in Figure 1.

\section{Morphology and porosity of nanofibrous scaffolds}

Electrospun PVA and PVA/chitosan scaffolds were observed by SEM in 3000 magnification. Figures 2 and 3 show the SEM micrographs of the electrospun PVA and PVA/chitosan scaffolds, respectively. As can be seen in Figure 3, in PVA/chitosan blend (weight ratio 90/10), the average fiber diameter was found to be $221 \mathrm{~nm}$ with a range of $94-410 \mathrm{~nm}$; while in PVA alone, the average fiber diameter was $744 \mathrm{~nm}$ with a range of 395-1105 nm (Figure 2). Similar observations have been made by Lin et $\mathrm{al}^{43}$ and Ignatova et $\mathrm{al}^{44}$ who investigated a series of PVA/chitosan blend nanofibrous membranes at different weight ratios and found a decrease in the average diameter of the nanofibers with increasing the chitosan content.

It should be noted that in electrospinning, the fiber diameter is dependent on the viscosity and charge of the solution.

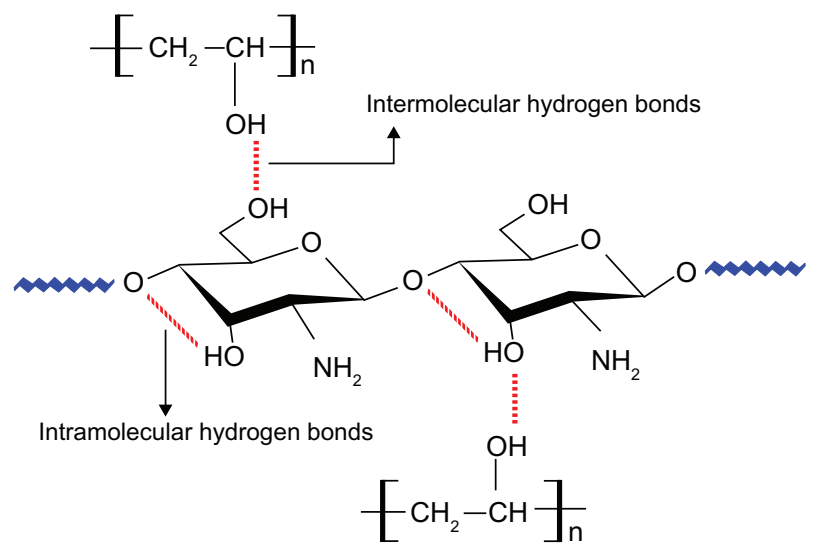

Figure I The schematic of intermolecular and intramolecular hydrogen bonds that occurred after blending PVA with chitosan.

Abbreviation: PVA, polyvinyl alcohol.
Typically, when the viscosity is increased, the diameter is increased proportionally. Chitosan affects not only the viscosity but also the charge density at the surface of the ejected jet through its cationic polyelectrolytic property. It increases the charge density at the surface of the jet which in turn increases the elongation force and decreases the diameter of the fiber. ${ }^{45}$

When the grayscale image was converted to binary form by ImageJ software, various layers of nanofibers could be seen by applying different thresholds. Figure 4 shows the configuration of histograms of PVA and PVA/chitosan nanofibrous scaffolds. As previously described by Ghasemi-Mobarakeh et al, ${ }^{34}$ the results are not dependent upon the magnification or histogram of images. Various layers in most image magnifications and histograms allow calculation of porosity.

Analysis was performed on various layers of nanofibers by application of thresholds (see Figure 5). Threshold 1 eliminated the upper layer, and so, the surface layers were captured, a representative sum of surface and middle layers was captured using threshold 2, and threshold 3 was used for capture of all of the visible layers.

After converting the original image to various binary images, the porosity of each binary image was calculated using the mean intensity of micrographs ${ }^{34}$ using equation 3 :

$$
P=\left(1-\frac{n}{N}\right) \times 100
$$

where $n$ is the number of white pixels, $N$ is the total number of pixels in binary image, and $P$ is the porosity of binary image. The porosity of binary images with thresholds of 1 , 2 , and 3 is presented as P1, P2, and P3, and the results of porosity calculations of various binary images with different thresholds are shown in Table 1.

Interestingly, it was shown that the porosity of the same layers in the scaffolds fabricated with PVA and PVA/chitosan blend did not differ significantly. However, the pore morphologies were different. As mentioned above, PVA solution yielded fibers with relatively large diameters, which formed pores larger than those formed by small-diameter PVA/chitosan fibers. The numbers of pores were also different. PVA/ chitosan scaffolds had a higher number of pores.

It should be noted that scaffold porosity and pore morphology are important for many tissue engineering applications as they allow migration of the cells and growth of blood vessels across the scaffold and ensure effective exchange of nutrients and waste products between the cells and their microenvironment. Here, we proposed and used an effective method for measurement of porosity at different layers of a scaffold. 

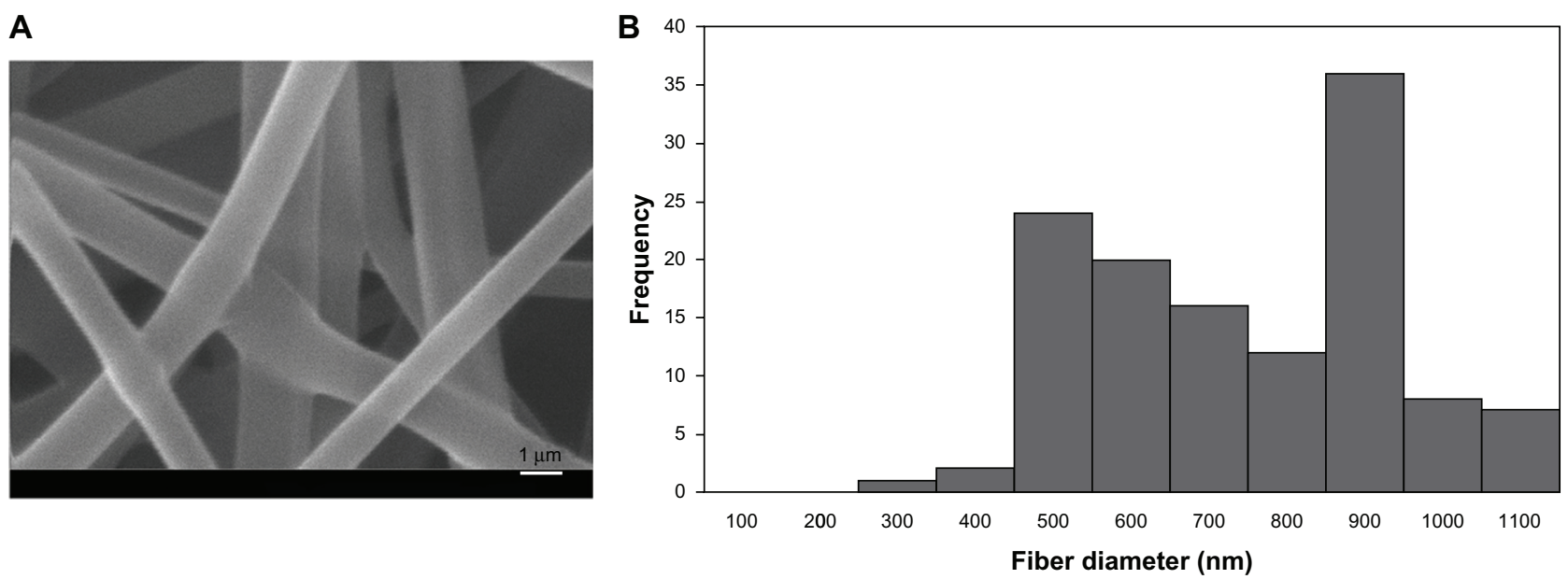

Figure 2 (A) SEM micrograph of electrospun PVA fibers; (B) fiber diameter distribution of PVA fibers. Abbreviations: SEM, scanning electron micrograph; PVA, polyvinyl alcohol.

\section{Chemical bonding}

FTIR spectroscopy was used to assess the chemical groups of the polymers. Figure 6 shows the FTIR spectra of PVA, chitosan, and PVA/chitosan blend. In Figure 6B, for the chitosan sample, the major characteristic peaks around 844 and $1151 \mathrm{~cm}^{-1}$ related to the saccharide structure (as the repeating unit of chitosan) are clearly observable. ${ }^{46,47}$ In addition, the strong absorption peaks at 1740,1480, and $1346 \mathrm{~cm}^{-1}$ are shown, which are characteristic of chitosan and have been reported as amide I, II, and III peaks, respectively. The sharp peaks at 1382 and $1417 \mathrm{~cm}^{-1}$ could be assigned to the $\mathrm{CH}_{3}$ symmetrical deformation mode. Also, the broad peaks at 1081 and $1122 \mathrm{~cm}^{-1}$ indicate the $\mathrm{C}-\mathrm{O}$ stretching vibration in chitosan, and another broad peak at $3447 \mathrm{~cm}^{-1}$ is caused by amine $\mathrm{N}-\mathrm{H}$ symmetrical vibration. The peak observed at around $2947 \mathrm{~cm}^{-1}$ is due to the typical $\mathrm{C}-\mathrm{H}$ stretch vibrations. ${ }^{48}$ In Figure 6A, all major peaks related to hydroxyl and acetate groups are shown in the FTIR spectrum of PVA. More specifically, the broad band observed between 3550 and $3200 \mathrm{~cm}^{-1}$ is associated with the $\mathrm{O}-\mathrm{H}$ stretch from the intermolecular and intramolecular hydrogen bonds. The vibrational band observed between 2840 and $3000 \mathrm{~cm}^{-1}$ is the result of the $\mathrm{C}-\mathrm{H}$ stretch from alkyl groups and the peaks between 1730 and $1680 \mathrm{~cm}^{-1}$ are due to the $\mathrm{C}=\mathrm{O}$ and $\mathrm{C}-\mathrm{O}$ stretches from the remaining acetate groups in PVA (saponification reaction of polyvinyl acetate). ${ }^{49-51}$

Moreover, the FTIR spectra of the PVA/chitosan fibers (Figure 6C) are typical of the characteristic peaks of PVA and chitosan except those related to the ionization of the primary amino groups of chitosan. These peaks are shown at 1408 and $1548-1560 \mathrm{~cm}^{-1}$. Formation of the $1552-1558 \mathrm{~cm}^{-1}$ peak is due to the symmetric deformation of $-\mathrm{NH}_{3}{ }^{+}$groups
A

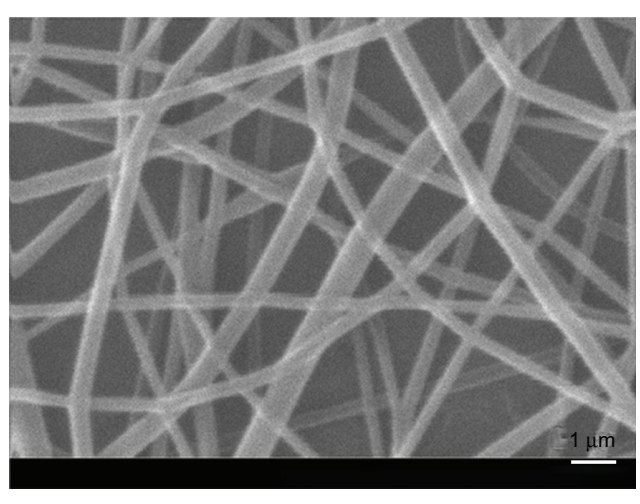

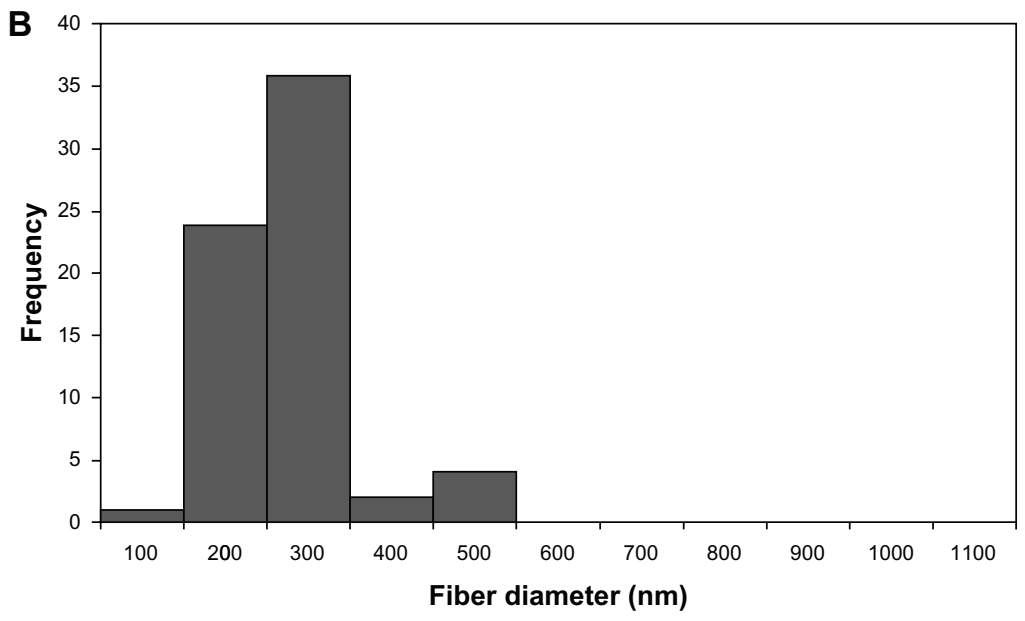

Figure 3 (A) SEM micrograph of electrospun PVA/chitosan nanofibers; (B) fiber diameter distribution of PVA/chitosan nanofibers. Abbreviations: SEM, scanning electron micrograph; PVA, polyvinyl alcohol. 

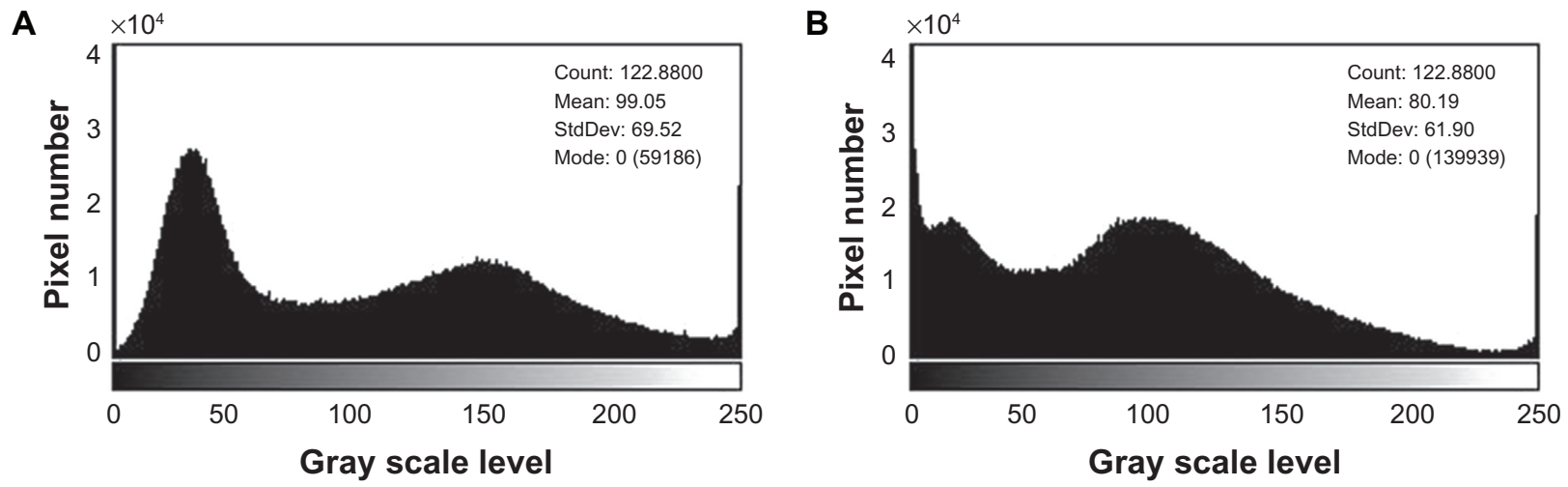

Figure 4 (A) Image histogram of PVA fibers; (B) image histogram of PVA/chitosan nanofibers. Abbreviation: PVA, polyvinyl alcohol.

and the peak at $1408 \mathrm{~cm}^{-1}$ is the result of carboxylic acid. The peaks shown at $1700-1725 \mathrm{~cm}^{-1}$ are characteristic of carboxylic acid dimers. ${ }^{10}$

\section{Swelling behavior}

Fluid uptake is an important parameter, which influences the chemical and physical characteristics of the scaffolds after and prior to cell seeding. Herein, swelling experiments were
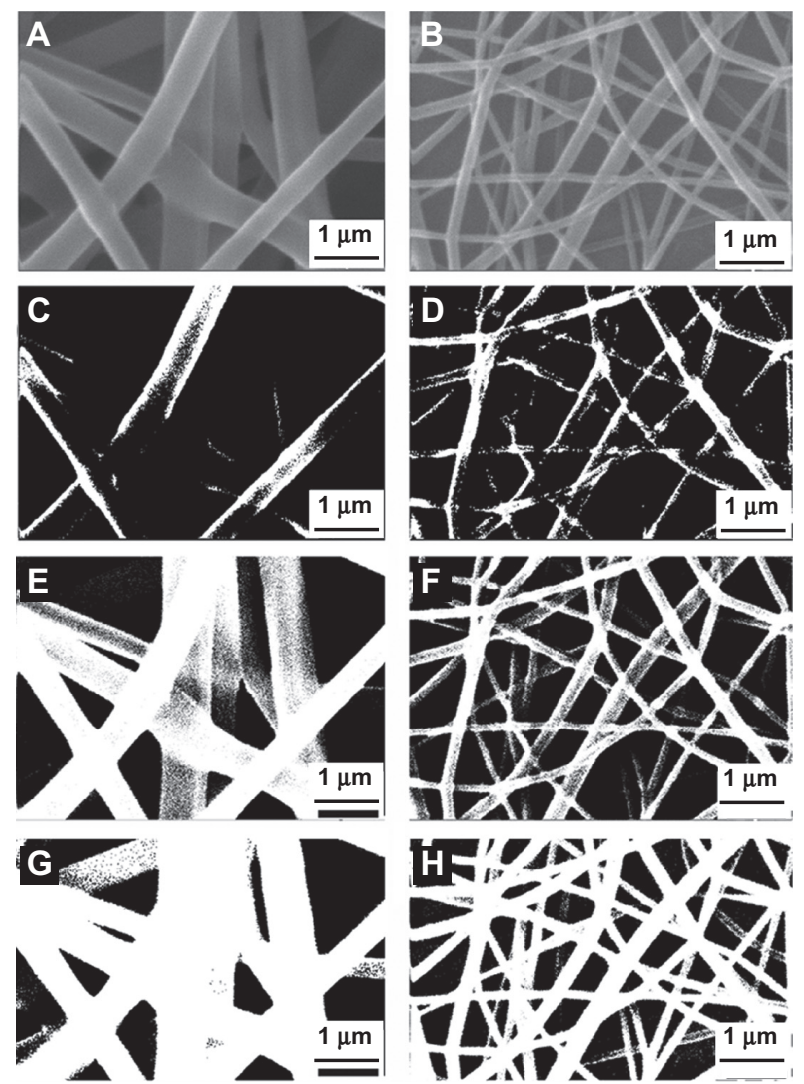

Figure $\mathbf{5}$ Various binary images with different thresholds for PVA and PVA/chitosan samples. (A) and (B) original images; (C) and (D) binary images with threshold I; (E) and (F) binary images with threshold 2; $(\mathbf{G})$ and $(\mathbf{H})$ binary images with threshold 3. performed after cross-linking of PVA and PVA/chitosan with GA vapor. A representative fluid uptake behavior is shown in Figure 7 for PVA and PVA/chitosan cross-linked scaffolds. The results revealed that chitosan strongly influences the swelling volume of the scaffold and decreases it from $450 \%$ (for PVA scaffold) to $300 \%$ (for PVA/chitosan scaffold). The reduced swelling volume could be attributed to a more rigid network formed by the inter- and intrapolymer reactions and also to the reduction of hydrophilic groups in PVA/chitosan blend. The latter is due to chitosan amine groups being more reactive to GA than to hydroxyls of PVA. This observation is in agreement with previous studies which reported that chitosan decreases the swelling rate when blended with PVA; the degree of reduction depends on factors such as weight ratio of the components, $\mathrm{pH}$, temperature, and so on. ${ }^{36,48-51}$

\section{Degradation behavior}

Degradation is the process through which useful physicochemical properties of the polymers are lost. This can include loss of polymer mass through mechanisms such as solvation and depolymerization. Degradation behavior of PVA and PVA/chitosan nanofibrous scaffolds using the PBS immersion method is shown in Figure 8. It was clearly observed that the degradation rate of PVA/chitosan scaffolds was much slower than PVA samples. This could be due to higher density of chemical cross-linking between GA and amine groups of chitosan and leads to slower depolymerization. ${ }^{52,53}$

Table I Porosity measurement of binary images of PVA and PVA/chitosan with various thresholds

\begin{tabular}{lllll}
\hline Sample & Magnification & PI & P2 & P3 \\
\hline PVA & 3000 & 89.3333 & 61.9190 & 34.9492 \\
PVA/chitosan & 3000 & 81.4113 & 59.8913 & 56.2653 \\
\hline
\end{tabular}

Abbreviation: PVA, polyvinyl alcohol. 


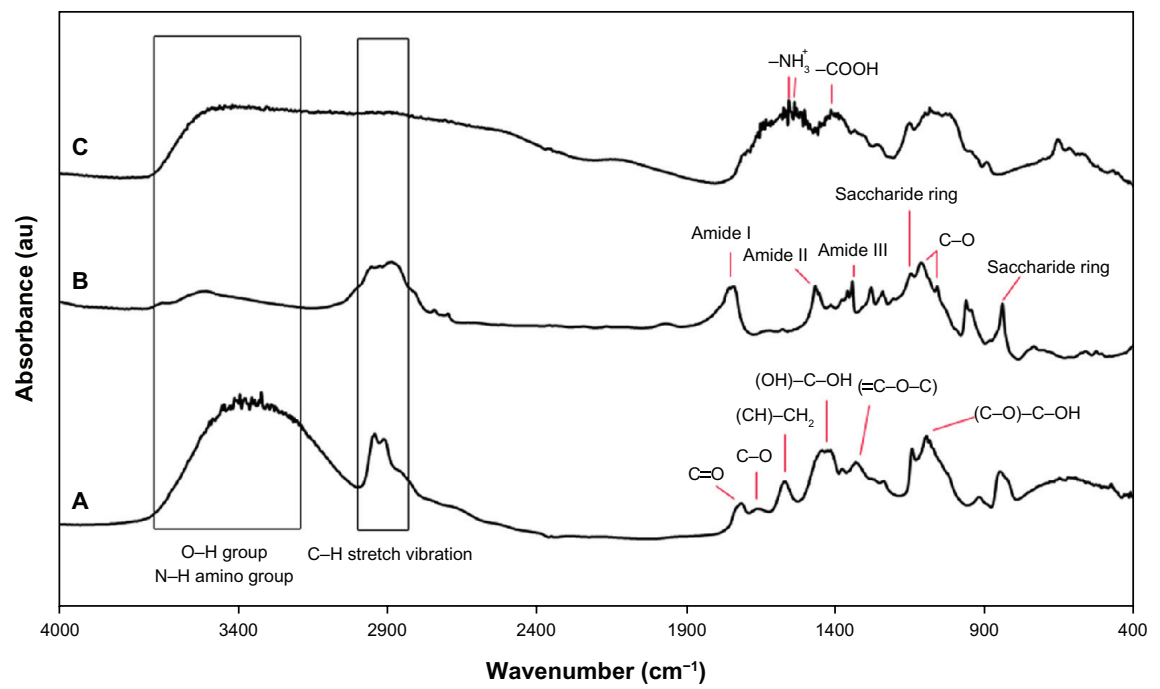

Figure 6 FTIR analysis of (A) PVA; (B) chitosan; (C) PVA/chitosan samples. Abbreviations: FTIR, Fourier transform infrared; PVA, polyvinyl alcohol.

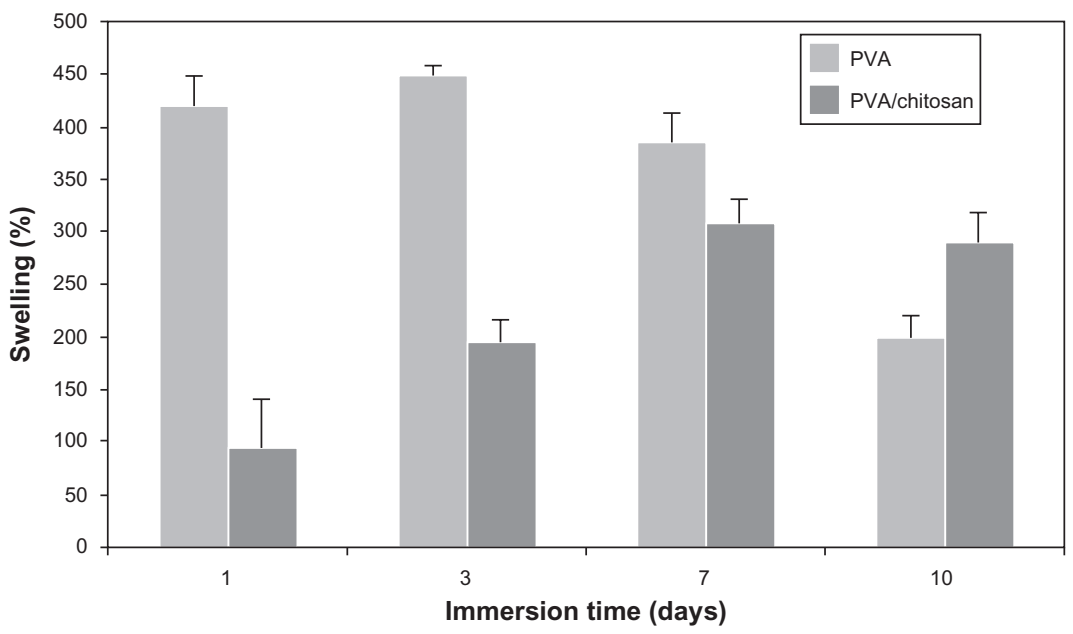

Figure 7 Swelling behavior of PVA and PVA/chitosan samples in different time intervals. Abbreviation: PVA, polyvinyl alcohol.

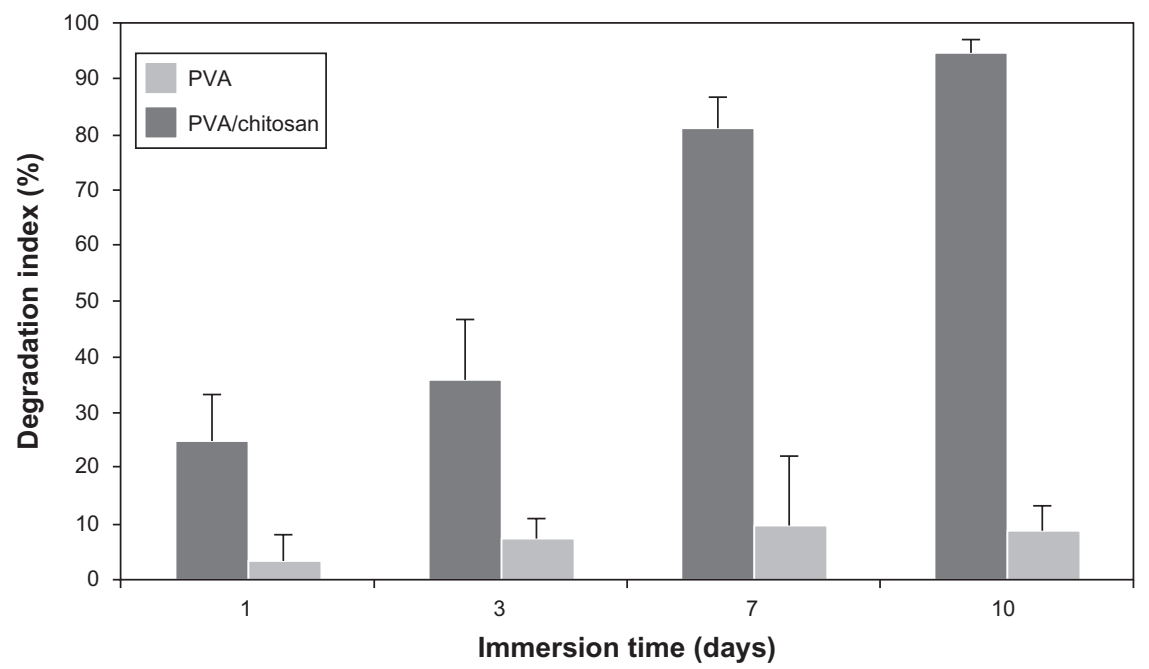

Figure 8 Degradation behavior of PVA and PVA/chitosan samples in different time intervals. Abbreviation: PVA, polyvinyl alcohol. 


\section{Viability of the nerve cells}

In this study, MTT assay was performed to evaluate the cell proliferation rate of PC12 nerve cells on the PVA and PVA/chitosan nanofibrous scaffolds. As shown in Figure 9, the proliferation of cells on PVA/chitosan scaffolds was higher than on PVA scaffolds, indicating that the chitosan
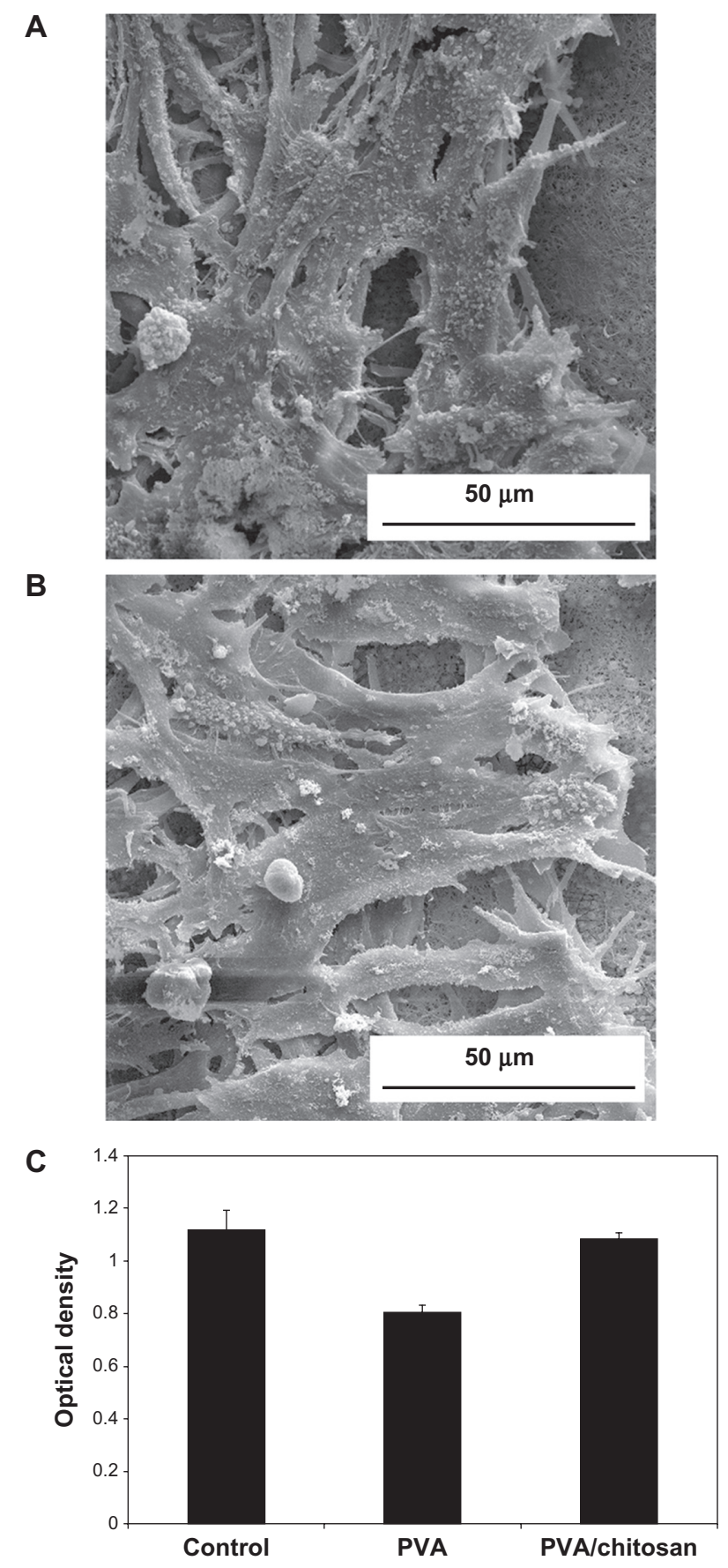

Figure 9 (A) and (B) Attachment of $\mathrm{PCI} 2$ nerve cells into PVA/chitosan nanofibrous scaffolds; (C) MTT analysis of PVA and PVA/chitosan samples.

Abbreviations: PVA, polyvinyl alcohol; MTT, 3-(4,5-Dimethylthiazol-2-yl)-2,5diphenyltetrazolium bromide. might have accelerated the proliferation rate of PC12 nerve cells. This could be attributed to the higher surface amine and the lower water contents of the swollen PVA/chitosan scaffold. In addition, it has been shown that fiber diameter can influence cell adhesion, proliferation, and migration. For example, it was shown by Christopherson et al that smaller fiber diameters favor better neural cell attachment and proliferation of cells cultured on non-woven electrospun fiber meshes..$^{54}$

\section{Conclusion}

In this study, polyvinyl alcohol (PVA) fibers were modified through blending with chitosan and electrospun to fabricate a nanofibrous scaffold. The PVA/chitosan scaffolds have been found to exhibit physicochemical and biological properties which, compared with PVA scaffolds, better meet the requirements of nerve cells.

\section{Acknowledgments}

The authors thank the many colleagues and collaborators who have made a vast contribution to this area of research.

\section{Disclosure}

The authors declare no conflict of interest in relation to this work.

\section{References}

1. Samadikuchaksaraei A. Scientific and industrial status of tissue engineering. Afr Biotechnol. 2007;6(25):2897-2909.

2. Chew SY, Mi R, Hoke A, Leong KW. The effect of the alignment of electrospun fibrous scaffolds on Schwann cell maturation. Biomaterials. 2008;29(6):653-661.

3. Bhattarai N, Edmondson D, Veiseh O, Matsen FA, Zhang M. Electrospun chitosan-based nanofibers and their cellular compatibility. Biomaterials. 2005;26(31):6176-6184.

4. Cao H, Liu T, Chew SY. The application of nanofibrous scaffolds in neural tissue engineering. Adv Drug Deliv Rev. 2009;61(12): 1055-1064.

5. Yim EK, Reano RM, Pang SW, Yee AF, Chen CS, Leong KW. Nanopatterninduced changes in morphology and motility of smooth muscle cells. Biomaterials. 2005;26(26):5405-5413.

6. Lee J, Cuddihy MJ, Kotov NA. Three-dimensional cell culture matrices: state of the art. Tissue Eng Part B Rev. 2008;14(1):61-86.

7. Yu L, Leipzig N, Shoichet M. Promoting neuron adhesion and growth. Mater Today. 2008;11(5):36-43.

8. Ma PX, Zhang R. Synthetic nano-scale fibrous extracellular matrix. J Biomed Mater Res. 1999;46(1):60-72.

9. Yang F, Xu CY, Kotaki M, Wang S, Ramakrishna S. Characterization of neural stem cells on electrospun poly(L-lactic acid) nanofibrous scaffold. J Biomater Sci Polym Ed. 2004;15(12):1483-1497.

10. Li D, Xia Y. Electrospinning of nanofibers: reinventing the wheel? $A d v$ Mater. 2004;16(14):1151-1170.

11. Reneker Darrell H, Chun I. Nanometre diameter fibres of polymer, produced by electrospinning. Nanotechnology. 1996;7(3): 216-223. 
12. Jain KK. Role of nanotechnology in developing new therapies for diseases of the nervous system. Nanomedicine. 2006;1:9-12.

13. Liao S, Li B, Ma Z, Wei H, Chan C, Ramakrishna S. Biomimetic electrospun nanofibers for tissue regeneration. Biomed Mater. 2006;1(3): R45-R53.

14. Cheng M, Deng J, Yang F, Gong Y, Zhao N, Zhang X. Study on physical properties and nerve cell affinity of composite films from chitosan and gelatin solutions. Biomaterials. 2003;24(17): 2871-2880.

15. Sanders EH, Kloefkorn R, Bowlin GL, Simpson DG, Wnek GE. Two-phase electrospinning from a single electrified jet: microencapsulation of aqueous reservoirs in poly(ethylene-co-vinylacetate) fibers. Macromolecules. 2003;36(11):3803-3805.

16. Ahmed I, Liu HY, Mamiya PC, et al. Three-dimensional nanofibrillar surfaces covalently modified with tenascin-C-derived peptides enhance neuronal growth in vitro. J Biomed Mater Res A. 2006;76(4): 851-860.

17. Bellamkonda RV. Peripheral nerve regeneration: an opinion on channels, scaffolds and anisotropy. Biomaterials. 2006;27(19):3515-3518.

18. Bini TB, Gao S, Wang S, Ramakrishna S. Poly(1-lactide-co-glycolide) biodegradable microfibers and electrospun nanofibers for nerve tissue engineering: an in vitro study. J Mater Sci. 2006;41(19): 6453-6459.

19. Ito S, Wang W, Tsuneo O, et al. Efficacy of chitosan nanofiber mesh as a scaffold for regenerating nerve tissue. Kichin Kitosan Kenkyu. 2006; 12:194-195.

20. Patel S, Kurpinski K, Quigley R, et al. Bioactive nanofibers: synergistic effects of nanotopography and chemical signaling on cell guidance. Nano Lett. 2007;7(7):2122-2128.

21. Schnell E, Klinkhammer K, Balzer S, et al. Guidance of glial cell migration and axonal growth on electrospun nanofibers of poly[epsilon]-caprolactone and a collagen/poly-[epsilon]-caprolactone blend. Biomaterials. 2007;28(19):3012-3025.

22. Corey JM, Lin DY, Mycek KB, et al. Aligned electrospun nanofibers specify the direction of dorsal root ganglia neurite growth. J Biomed Mater Res A. 2007;83A(3):636-645.

23. Chen JP, Chang GY, Chen JK. Electrospun collagen/chitosan nanofibrous membrane as wound dressing. Colloids Surf A: Physicochem Eng Asp. 2008;313-314:183-188.

24. Sahoo S, Ouyang H, Goh JCH, Tay TE, Toh SL. Characterization of a novel polymeric scaffold for potential application in tendon/ligament tissue engineering. Tissue Eng. 2006;12(1):91-99.

25. Zheng-Qiu G, Jiu-Mei X, Xiang-Hong Z. The development of artificial articular cartilage - PVA-hydrogel. Biomed Mater Eng. 1998;8(2): 75-81.

26. Miyashita H, Shimmura S, Kobayashi H, et al. Collagen-immobilized poly (vinyl alcohol) as an artificial cornea scaffold that supports a stratified corneal epithelium. J Biomed Mater Res B Appl Biomater. 2006;76(1):56-63

27. Jafari J, Emami SH, Samadikuchaksaraei A, Bahar MA, Gorjipour F. Electrospun chitosan-gelatin nanofiberous scaffold: fabrication and in vitro evaluation. BioMed Mater Eng. 2011;21(2):99-112.

28. Emami SH, Abad AMA, Bonakdar S, Samadikuchaksaraei A, Bahar MA. Preparation and evaluation of chitosan-gelatin composite scaffolds modified with chondroitin-6-sulphate. Int J Mater Res. 2010;101(10): 1281-1285.

29. Zhang XF, Cao WL, Gong YD, et al. A method for the preparation of porous chitosan tube. China Patent. 2002:ZL 02149086. [Chinese].

30. Wang A, Ao Q, Cao W, et al. Fiber-based chitosan tubular scaffolds for soft tissue engineering: fabrication and in vitro evaluation. Tsinghua Sci Technol. 2005;10(4):449-453.

31. Dillon GP, Yu X, Sridharan A, Ranieri JP, Bellamkonda RV. The influence of physical structure and charge on neurite extension in a 3D hydrogel scaffold. J Biomater Sci Polym Ed. 1998;9(10): 1049-1069.
32. Wang X, Hu W, Cao Y, Yao J, Gu X. Dog sciatic nerve regeneration across a $30-\mathrm{mm}$ defect bridged by a chitosan/PGA artificial nerve graft. Brain. 2005;128(8):1897-1910.

33. Ciardelli G, Chiono V. Materials for peripheral nerve regeneration. Macromol Biosci. 2006;6(1):13-26.

34. Semnani D, Lattifi M. Effect of yarn appearance on apparent quality of weft knitted fabric. J Text I. 2005;96(5):295-301.

35. Ghasemi-Mobarakeh L, Semnani D, Morshed M. A novel method for porosity measurement of various surface layers of nanofibers mat using image analysis for tissue engineering applications. J Appl Polym Sci. 2007;106(4):2536-2542.

36. de Souza Costa-Júnior E, Pereira MM, Mansur HS. Properties and biocompatibility of chitosan films modified by blending with PVA and chemically crosslinked. J Mater Sci Mater Med. 2009;20(2): 553-561.

37. Paipitak K, Pornpra T, Mongkontalang P, Techitdheer W, Pecharapa W. Characterization of PVA-chitosan nanofibers prepared by electrospinning. Procedia Eng. 2011;8:101-105.

38. Zheng H, Du Y, Yu J, Huang R, Zhang L. Preparation and characterization of chitosan/poly(vinyl-alcohol) blend fibers. J Appl Polym Sci. 2001;80(13):2558-2565.

39. Miya M, Iwamoto R, Mima S. FT-IR study of intermolecular interactions in polymer blends. J Polym Sci Polym Phys Ed. 1984;22(6): 1149-1151.

40. Nakatsuka S, Andrady AL. Permeability of vitamin B-12 in chitosan membranes. Effect of crosslinking and blending with poly(vinyl alcohol) on permeability. J Appl Polym Sci. 1992;44(1):17-28.

41. Cho YW, Han SS, Ko SW. PVA containing chito-oligosaccharide side chain. Polym. 2000;41(6):2033-2039.

42. Jin L, Bai R. Mechanisms of lead absorption on chitosan/PVA hydrogel beads. Langmuir. 2002;18:9765-9770.

43. Lin T, Fang J, Wang H, Cheng T, Wang X. Using chitosan as a thickener for electrospinning dilute PVA solutions to improve fibre uniformity. Nanotechnology. 2006;17(15):3718

44. Ignatova M, Starbova K, Markova N, Manolova N, Rashkov I. Electrospun nano-fibre mats with antibacterial properties from quaternised chitosan and poly(vinyl alcohol). Carbohydr Res. 2006;341(12): 2098-2107.

45. Sajeev US, Anand KA, Menon D, Nair S. Control of nanostructures in PVA, PVA/chitosan blends and PCL through electrospinning. Bull Mater Sci. 2008;31(3):343-351.

46. Mansur HS, Costa HS. Nanostructured poly(vinyl alcohol)/bioactive glass and poly(vinyl alcohol)/chitosan/bioactive glass hybrid scaffolds for biomedical applications. Chem Eng J. 2008;137(1):72-83.

47. Costa Jr EDS, Mansur HS. Preparation and characterization of chitosan/poly(vinylalcohol)blend chemically crosslinked by glutaraldehyde for tissue engineering application. Química Nova. 2008;31: 1460-1466.

48. Don TM, King CF, Chiu WY, Peng CA. Preparation and characterization of chitosan-g-poly(vinyl alcohol)/poly(vinyl alcohol) blends used for the evaluation of blood-contacting compatibility. Carb Polym. 2006; 63(3):331-339.

49. Wang T, Turhan M, Gunasekaran S. Selected properties of $\mathrm{pH}$-sensitive, biodegradable chitosan-poly(vinyl alcohol) hydrogel. Polym Int 2004;53(7):911-918.

50. Shigemasa Y, Matsuura H, Sashiwa H, Saimoto H. Evaluation of different absorbance ratios from infrared spectroscopy for analyzing the degree of deacetylation in chitin. Int J Bio Macromol. 1996;18(3): 237-242.

51. Mansur HS, Sadahira CM, Souza AN, Mansur AAP. FTIR spectroscopy characterization of poly (vinyl alcohol) hydrogel with different hydrolysis degree and chemically crosslinked with glutaraldehyde. Mater Sci Eng C. 2008;28(4):539-548.

52. Suh JK, Matthew HW. Application of chitosan-based polysaccharide biomaterials in cartilage tissue engineering: a review. Biomaterials. 2000;21(24):2589-2598. 
53. Berger J, Reist M, Mayer JM, Felt O, Peppas NA, Gurny R. Structure and interactions in covalently and ionically crosslinked chitosan hydrogels for biomedical applications. Eur J Pharm Biopharm. 2004; 57(1):19-34.
54. Christopherson GT, Song H, Mao HQ. The influence of fiber diameter of electrospun substrates on neural stem cell differentiation and proliferation. Biomaterials. 2009;30(4):556-564.

\section{Publish your work in this journal}

The International Journal of Nanomedicine is an international, peerreviewed journal focusing on the application of nanotechnology in diagnostics, therapeutics, and drug delivery systems throughout the biomedical field. This journal is indexed on PubMed Central, MedLine, CAS, SciSearch ${ }^{\circledR}$, Current Contents ${ }^{\circledR} /$ Clinical Medicine,
Journal Citation Reports/Science Edition, EMBase, Scopus and the Elsevier Bibliographic databases. The manuscript management system is completely online and includes a very quick and fair peer-review system, which is all easy to use. Visit http://www.dovepress.com/ testimonials.php to read real quotes from published authors.

Submit your manuscript here: http://www.dovepress.com/international-journal-of-nanomedicine-journal 